\section{RMD Open}

Rheumatic \&

Musculoskeletal Diseases

\title{
Antiphospholipid antibodies in COVID-19: a meta-analysis and systematic review
}

\author{
Muhanad Taha (D) , Lobelia Samavati
}

To cite: Taha M, Samavati L. Antiphospholipid antibodies in COVID-19: a meta-analysis and systematic review. RMD Open 2021;7:e001580. doi:10.1136/ rmdopen-2021-001580

- Additional supplemental material is published online only. To view, please visit the journal online (http://dx.doi.org/10. 1136/rmdopen-2021-001580).

Received 13 January 2021 Revised 11 April 2021 Accepted 13 April 2021

Check for updates

C) Author(s) (or their employer(s)) 2021. Re-use permitted under CC BY-NC. No commercial re-use. See rights and permissions. Published by BMJ.

Department of Pulmonary/ Critical Care, Wayne State University, Detroit, Michigan, USA

\section{Correspondence to} Dr Lobelia Samavati; Isamavat@med.wayne.edu

\section{ABSTRACT}

Background Many studies reported high prevalence of antiphospholipid antibodies (aPL) in patients with COVID-19 raising questions about its true prevalence and its clinical impact on the disease course.

Methods We conducted a meta-analysis and a systematic review to examine the prevalence of aPL and its clinical impact in patients with COVID-19.

Results 21 studies with a total of 1159 patients were included in our meta-analysis. Among patients hospitalised with COVID-19, the pooled prevalence rate of one or more aPL (IgM or IgG or IgA of anticardiolipin (aCL) or anti-B2 glycoprotein (anti-B2 GPI) or antiphosphatidylserine/ prothrombin, or lupus anticoagulant (LA)) was $46.8 \%$ (95\% $\mathrm{Cl} 36.1 \%$ to $57.8 \%$ ). The most frequent type of aPL found was LA, with pooled prevalence rate of $50.7 \%(95 \% \mathrm{Cl}$ $34.8 \%$ to $66.5 \%)$. Critically ill patients with COVID-19 had significantly higher prevalence of aCL (IgM or IgG) $(28.8 \%$ vs $7.10 \%, p<0.0001)$ and anti-B2 GPI (IgM or IgG) $(12.0 \%$ vs $5.8 \%, p<0.0001$ ) as compared with non-critically ill patients. However, there was no association between aPL positivity and mean levels of $\mathrm{C}$ reactive protein (mean difference was $32(95 \% \mathrm{Cl}-15$ to 79$), \mathrm{p}=0.18)$, D-dimer (mean difference was 34 (95\% Cl -194 to 273), $\mathrm{p}=0.77$ ), mortality (1.46 (95\% Cl 0.29 to 7.29$), p=0.65)$, invasive ventilation (1.22 (95\% $\mathrm{Cl} 0.51$ to 2.91$), \mathrm{p}=0.65)$ and venous thromboembolism (1.38 (95\% $\mathrm{Cl} 0.57$ to 3.37$)$, $\mathrm{p}=0.48$ ).

Conclusions aPLs were detected in nearly half of patients with COVID-19, and higher prevalence of aPL was found in severe disease. However, there was no association between aPL positivity and disease outcomes including thrombosis, invasive ventilation and mortality. However, further studies are required to identify the clinical and pathological role of aPL in COVID-19.

\section{INTRODUCTION}

Hypercoagulability is one of the striking features of COVID-19. In a recent large study, risk of venous thromboembolism in patients with COVID-19 was $16 \%$, while risk of arterial thrombosis was $11.1 \% .^{1}$ Others documented cerebral ischaemic infarcts in these subjects. ${ }^{2}$ In addition to macrothrombi, several autopsy studies of patients with COVID-19 revealed features of microangiopathy with

\section{Key messages}

What is already known about this subject?

- Antiphospholipid antibodies (aPLs) were frequently reported in patients with COVID-19. However, its true prevalence and its clinical impacts are unknown.

What does this study add?

- We conducted the largest meta-analysis to date examining the prevalence and the clinical impact of aPL on the clinical features of patients with COVID-19 Our significant findings are: (1) nearly half of patients with COVID-19 were positive for one of the aPL. (2) Most frequently reported aPL was LA. (3) aPLs were significantly more frequently reported in critically ill patients, and (4) aPLs were not significantly associated with disease outcomes like venous thrombosis, invasive ventilation and mortality.

How might this impact on clinical practice or further developments?

- The frequently reported aPL in patients with COVID-19, especially in critically ill patients, raises questions about its role in the pathogenesis of the disease.

microthrombi in various organs including, lung, kidney, heart, skin and prostate. ${ }^{34}$ Case fatality appears to be determined by formation of vascular thrombi in association with progressive severe endothelial injury in COVID-19 infected subjects. ${ }^{5}$ The clinical features related to vasculopathy and thromboembolism in patients with COVID-19 are wide ranging from asymptomatic with mild elevation of D-dimer to severe organ dysfunction due to macrothrombi and microthrombi. ${ }^{5}$ The pathogenesis of hypercoagulability in COVID-19 is not fully understood. Yet, SARS-CoV-2 mediated coagulopathy appears to have distinct features, such as normal to elevated fibrinogens, elevated D-Dimers, normal platelets and mild prolonged activated partial thromboplastin time. ${ }^{6}$ To identify the possible causes of macroangiopathy and microangiopathy in this disease, 
numerous studies evaluated the potential role of anticardiolipin antibodies (aPLs). ${ }^{2-26}$ In the current study, we conducted a meta-analysis and a systematic review to investigate if there is an association between COVID-19 and aPL.

\section{MATERIAL AND METHOD Aims and overview}

The aims of this meta-analysis study are to describe the actual prevalence of aPL in patients with COVID-19 and to describe the potential clinical impact of positive aPL on the disease outcome.

\section{Search strategy and study selection}

The systematic review and meta-analysis were conducted adhering to Preferred Reporting Items for Systematic Reviews and Meta-Analyses (PRISMA) guidelines. ${ }^{27} \mathrm{We}$ identified articles through a search of PubMed, MEDLINE, Embase, Web of Science and Google Scholar from 31 December 2019 to 15 October 2020. The following search terms were used: "COVID-19", "SARS-COV-2", "Antiphospholipid", "Anticardiolipin", "Lupus anticoagulant" and "Anti-B2 glycoprotein". Studies reporting aPL in patients with COVID-19 were extracted. We also reviewed the references of each study to identify further related articles for analysis. There was no language restriction. The two investigators independently performed the search and determined the eligibility of studies according to the criteria mentioned below. Data extracted from the included studies using a data extraction form developed in MS Excel (online supplemental table 1). Selection results have been reported according to the PRISMA flow chart (figure 1).

\section{Inclusion and exclusion criteria}

All studies met the following criteria: (1) subjects were adults and diagnosed with COVID-19 based on RT-PCR or serum serological testing, (2) any of the following aPL tests performed: IgM or IgG of anticardiolipin (aCL) or anti-32 glycoprotein (anti-32 GPI) or lupus anticoagulant (LA) or antiphosphatidylserine/ prothrombin (aPS/PT), (3) study sample was larger than 10 and (4) subjects had been randomly selected. We excluded articles with $<10$ subjects, case reports and paediatric population ( $<18$ years old). In addition, we excluded articles with non-randomly selected population (eg, COVID-19 with cerebrovascular accidents or thromboembolism).

\section{Antiphospholipid assay}

aCL, anti-B2 GPI and aPS/PT were detected in all studies using either ELISA or chemiluminescent immunoassay. The cut-off value for positive result was $>20 \mathrm{U}$ for aCL and anti- 32 GPI and $>30 \mathrm{U}$ for aPS/PT (except for study, ${ }^{26}$ the cut-off value for positive result was $>15 \mathrm{U}$ for aCL and >8U for anti-B2 GPI). Seven studies ${ }^{9}$ 13-15 181922 did not mention the cut-off value for positive result. We considered positive titre according to the authors' definition.

All studies mentioned the diagnostic method for detecting LA except two studies. ${ }^{21}{ }^{24}$ The rest of the studies detected LA using dilute Russell's viper venom time (dRVVT) screen, mixing and confirm assay. Not all studies reported details about heparin dose. Among the studies for which LA testing was conducted and details about heparin dose were reported, $71 \%$ subjects were on prophylactic dose, while the rest received therapeutic dose.

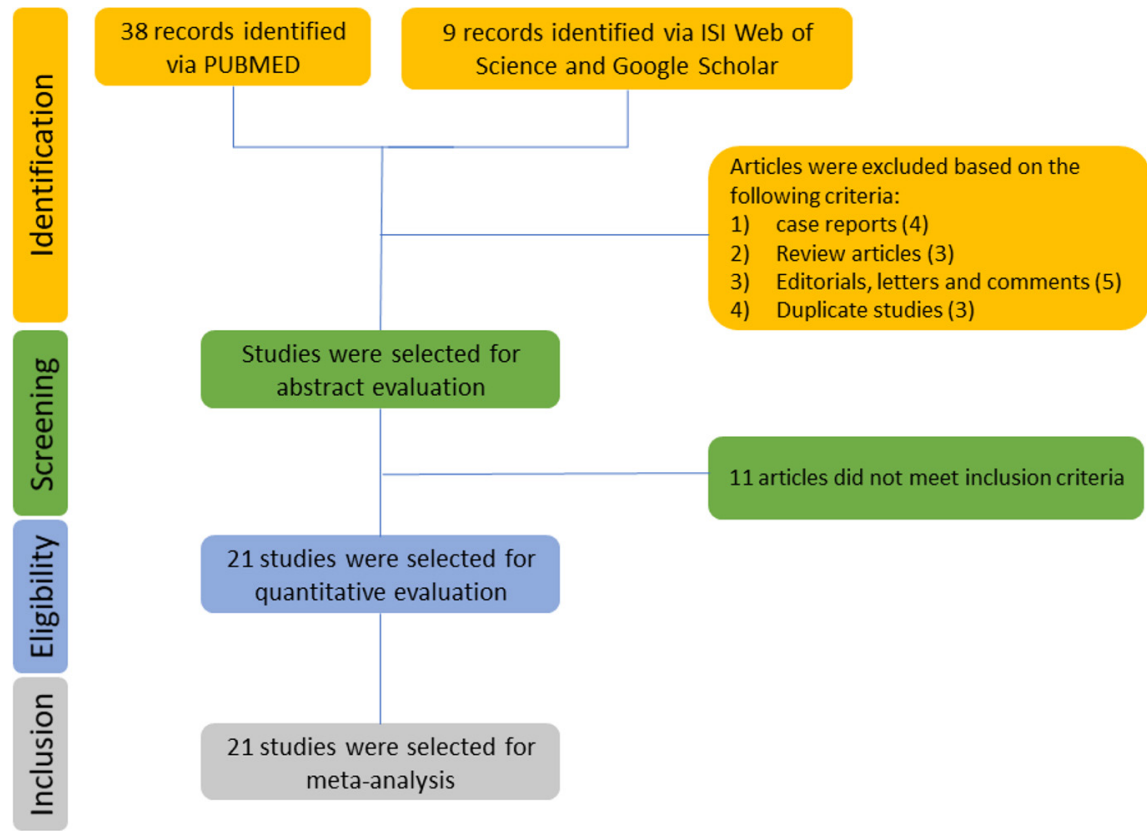

Figure 1 Searching and selection process. 


\section{Statistical analysis}

The statistical analysis was performed using Review Manager (V.5.4.1) provided by The Cochrane Collaboration. $\mathrm{P}$ value was two tailed, and the statistical significance set at $\leq 0.05$. Weighted pooled prevalence and 95\% CIs of aPL, aCL, LA and anti-32 GPI were calculated using a random-effects model. Weighted pooled prevalence of aPL, aCL, LA and anti-32 GPI was compared between intensive care unit (ICU) and non-ICU patients. All studies reported prevalence of aPL without a control group, so OR was not calculated. The association between aPL status and hospital outcomes (thrombosis, invasive ventilation and in-hospital mortality) was studied and expressed as OR with pertinent $95 \%$ CI. The association between aPL status and D-dimer and $\mathrm{C}$ reactive protein (CRP) was also examined and expressed as OR with pertinent 95\% CIs. D-dimer and CRP were described using mean and SD. Unit discordance for variables was resolved by converting all units to a standard measurement for that variable. A random-effects model was used to combine data. We assessed the heterogeneity in this meta-analysis by using the $\mathrm{I}^{2}$ statistical test. Assessment for publication bias was not done as less than 10 studies were included in each analysis so the power of the tests is too low to distinguish chance from real asymmetry.

\section{RESULTS}

\section{Study selection}

After searching database, we identified a total of 47 studies. After scanning the title/abstract, case reports, review articles, editorials, letters and comments, and duplicate studies were excluded. Further selection yielded to 32 potentially relevant studies that we accessed for further eligibility. Of these, 11 studies were excluded for the following reasons: paediatric population $<18$ years old, the study population was not randomly selected, and study sample was $<10$ subjects. Thus, 21 articles reflecting 1159 patients were included for analysis (online supplemental table 1). The searching and selection process is summarised in (figure 1).

\section{Study characteristics}

The selected articles ${ }^{2}$ 7-26 and extracted data are included in (online supplemental table 1). All the studies were either cross-sectional or retrospective observational studies. The frequency of aPL was reported in all studies. aPLs were reported in hospitalised patients, either critically ill patients or non-critically ill patients or both: 10 studies conducted on critically ill patients, ${ }^{2} 79101215181924251$ study in non-critically ill patients $^{21}$ and 10 studies in both. ${ }^{811} 1314202223262829$ We found nine studies were comparing patients with positive and negative aPL. ${ }^{7} 10131822242829$ SARS-CoV-2 infection was confirmed in all studies, and the predominate reported confirmation method was RT-PCR test. However, few studies,, 1115192025 did not specify which method was used to confirm SARS-CoV-2 infection. Information about when the laboratory testing for cardiolipin antibodies was performed as well as information about in-hospital treatment with antiviral, corticosteroids or heparin during hospitalisation was not available in most of the studies.

\section{Prevalence rates of aPL in patients with COVID-19}

In this analysis, we included the prevalence of antibodies for all studies. A total of 1159 patients (from 21 studies) hospitalised with COVID-19 and had one of the aPLs reported were included in this analysis. The pooled prevalence rate of one or more aPL (IgM or IgG of aCL or anti-32 GPI or aPS/PT, or LA) was $46.8 \%$ (95\% CI 36.1\% to $57.8 \%$ ) (table 1). The most frequent type of aPL found was LA, with pooled prevalence rate of $50.7 \%$ (95\% CI $34.8 \%$ to $66.5 \%$ ) (table 1 ). The pooled prevalence rate of aCL (IgM or IgG) and anti- 32 GPI (IgM or IgG) were $13.9 \%$ (95\% CI $7.5 \%$ to $24.1 \%$ ) and $6.7 \%$ (95\% CI $3.5 \%$ to $12.5 \%$ ), respectively (table 1 ). Four studies ${ }^{9} 172022$ reported double and triple aPL positivity. Pooled prevalence was $14.3 \%$ (95\% CI $4.0 \%$ to $40.3 \%$ ) for double aPL positivity and $6.1 \%$ (95\% CI $2.8 \%$ to $12.7 \%$ ) for triple aPL positivity. $\mathrm{I}^{2}$ test did not show significant heterogenicity between studies (table 1 ).

\section{Repeated antiphospholipid assay}

Two studies retested aPL in few patients with COVID-19 at later time point during hospitalisation. One study ${ }^{25}$ found that 9 out of 10 LA-positive patients were retested negative at later time. The other study ${ }^{28}$ retested aPL in six patients at multiple time-points and found that aPL

\begin{tabular}{|c|c|c|}
\hline Antibodies & Pooled prevalence of aPL $(95 \% \mathrm{Cl})(\%)$ & $I^{2}$ test \\
\hline$\geq \mathrm{aPL}$ & 46.8 (36.1 to 57.8 ) & $41.9 \%, p=0.29$ \\
\hline LA & 50.7 (34.8 to 66.5$)$ & $44.8 \%, p=0.11$ \\
\hline $\mathrm{aCL}(\lg M$ or $\lg G)$ & $13.9(7.5$ to 24.1$)$ & $23.6 \%, p=0.12$ \\
\hline anti-B2 GPI (IgM or lgG) & 6.7 (3.5 to 12.5$)$ & $0.0 \%, p=0.21$ \\
\hline Double positive aPL & $14.3(4.0$ to 40.3$)$ & $40 \%, p=0.32$ \\
\hline Triple positive aPL & $6.1(2.8$ to 12.7$)$ & $10.3 \%, p=0.25$ \\
\hline
\end{tabular}

$\mathrm{I}^{2}$ statistic describes the percentage of heterogeneity among the studies.

aCL, anticardiolipin; anti-B2 GPI, anti-B2 glycoprotein; $\mathrm{APL}$, anticardiolipin antibodies; LA, lupus anticoagulant. 


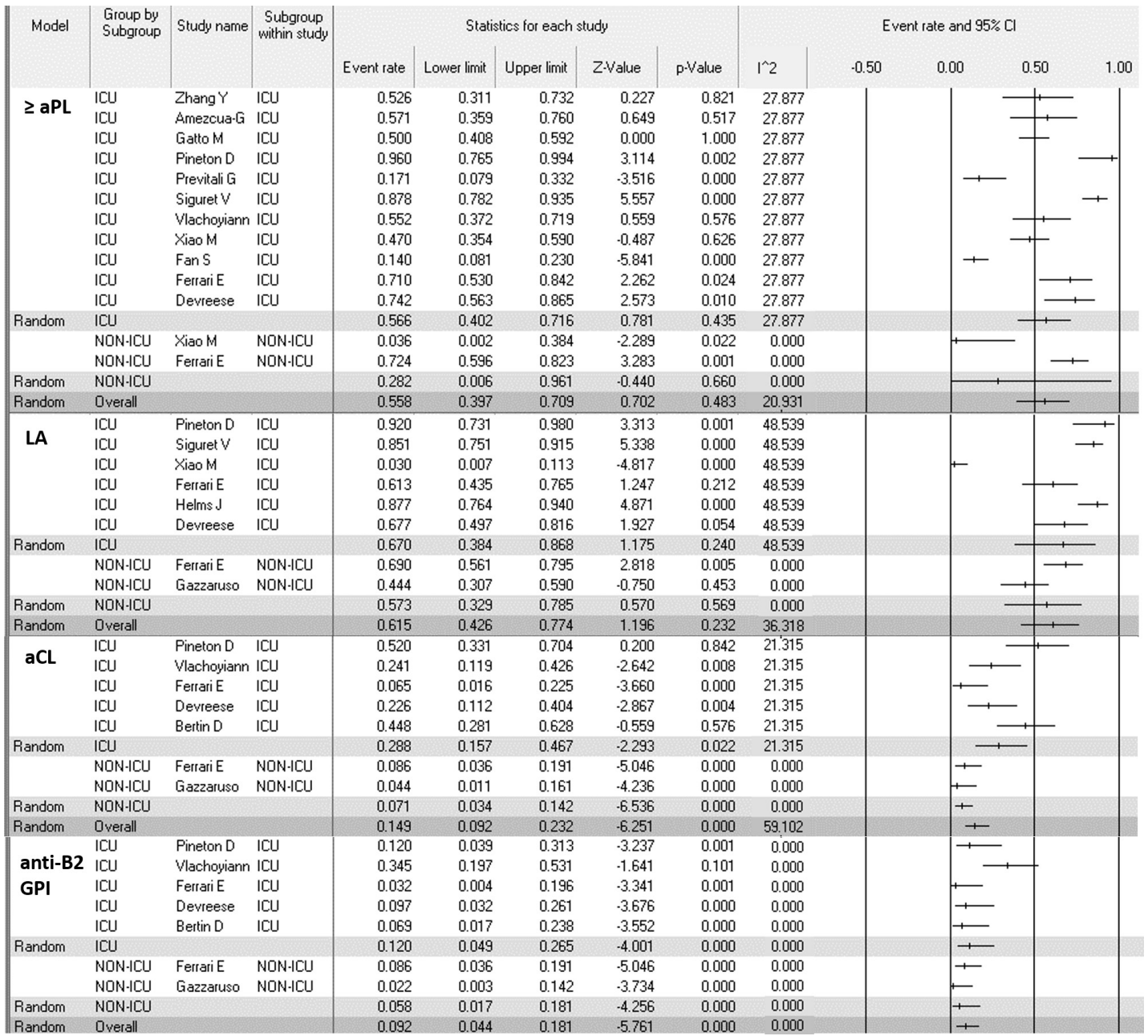

Figure 2 Forest plot on the prevalence of aPL in ICU versus non-ICU patients with COVID-19. aCL, anticardiolipin; anti-B2 GPI, anti-B2 glycoprotein; aPL, anticardiolipin antibodies; ICU, intensive care unit; LA, lupus anticoagulant.

levels, in general, reached a peak, which then declined over 3-4 week follow-up periods. These results suggest that aPL may be transiently elevated in patients with COVID-19.

\section{Prevalence of aPL in ICU versus non-ICU patients with COVID-19}

This meta-analysis found that critically ill patients with COVID-19 had significantly higher prevalence of aCL (IgM or IgG) $(28.8 \%$ vs $7.10 \%, \mathrm{p}<0.0001)$ and anti-32 GPI (IgM or $\operatorname{IgG})(12.0 \%$ vs $5.8 \%, \mathrm{p}<0.0001)$ as compared with non-critically ill patients (figure 2). However, there was no statistically significant difference in prevalence of LA or aPL in general between critically and non-critically ill patients with COVID-19 (figure 2). $\mathrm{I}^{2}$ test did not show significant heterogenicity between studies (figure 2).

\section{Outcomes of COVID-19 related hospitalisation in positive} versus negative aPL

Eight studies 28111416202225 examined the association of aPL positivity and hospital outcomes. Importantly, pooling data from these studies showed no association between aPL positivity and mean levels of CRP (mean difference was 32 (95\% CI -15 to 79$), p=0.18$ ) and D-dimer (mean difference was 34 (95\% CI -194 to 273 ), $\mathrm{p}=0.77$ ) (figure 3 ). $\mathrm{I}^{2}$ test did show significant heterogenicity between studies in CRP analysis $\left(\mathrm{I}^{2}=75 \%\right.$, $\mathrm{p}=0.008)$ but not in $\mathrm{D}$-dimer analysis $\left(\mathrm{I}^{2}=0.0 \%, \mathrm{p}=0.57\right)$.

Similarly, there was no association between aPL positivity and mortality (1.46 (95\% CI 0.29 to 7.29 ), $\mathrm{p}=0.65$ ), invasive ventilation ( 1.22 (95\% CI 0.51 to 2.91$), \mathrm{p}=0.65)$ and venous thromboembolism (1.38 (95\% CI 0.57 to 3.37 ), $\mathrm{p}=0.48$ ) (figures 4 and 5). There was significant heterogeneity between studies in mortality $\left(\mathrm{I}^{2}=74 \%, \mathrm{p}=0.004\right)$ and venous thromboembolism $\left(\mathrm{I}^{2}=68 \%, \mathrm{p}=0.003\right)$ but no heterogeneity in invasive ventilation analysis $\left(\mathrm{I}^{2}=19 \%\right.$, $\mathrm{p}=0.029$ ). The heterogeneity observed across studies can be explained by methodological differences like different type of aPL tested, different methods of testing and different positivity cut-off.

\section{DISCUSSION}

In this study, we conducted the largest meta-analysis to date examining the prevalence of aPL in COVID-19 and the association between aPL and disease severity. Our significant findings are: (1) nearly half of patients with COVID-19 were positive for one of the aPL; (2) most 
A. CRP

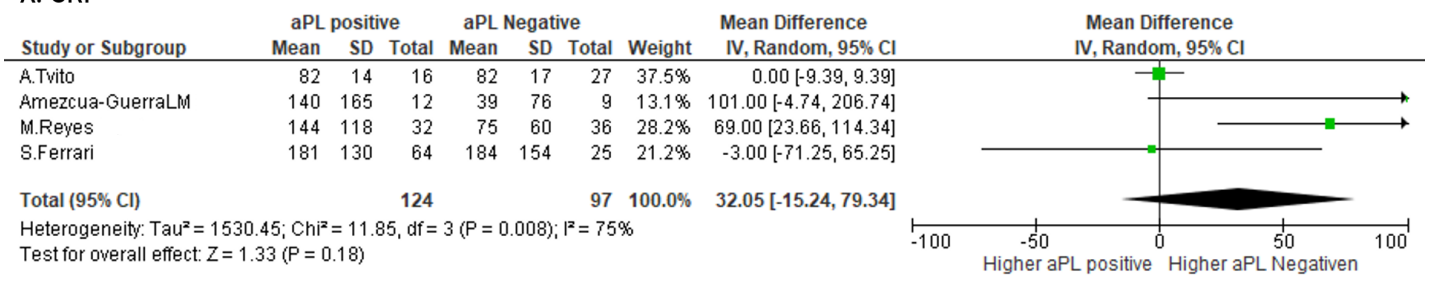

B. D-dimer

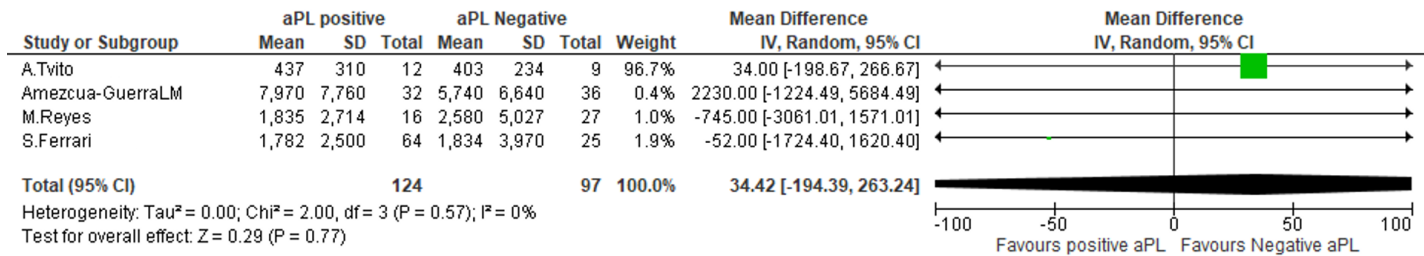

Figure 3 Forest plot on the mean difference of CRP (A) and D-dimer (B) in aPL-positive patients with COVID-19 compared with aPL-negative patients with COVID-19. aPL, anticardiolipin antibodies; CRP, C reactive protein.

frequently reported aPL was LA; (3) aCL and anti-B2 GPI were significantly more frequently reported in critically ill patients; and (4) aPLs were not significantly associated with disease severity.

The association between aPL and viral infections is widely acknowledged. ${ }^{30}$ We compared findings of this study with previous studies that examined the prevalence of aPL in other viral infections. ${ }^{30} 31$ We find that the prevalence of aCL was much lower, and the prevalence of LA was strikingly higher in our analysis related to SARS-CoV-2 compared with the ones previously reported in other viral infections. ${ }^{31}$ For instance, a previous metaanalysis ${ }^{31}$ reported very high prevalence of aCL in HIV (56\%), Epstein-Barr virus (EBV) $(50 \%)$ and hepatitis C virus (HCV) (21\%) compared with the aCL prevalence in our analysis $(13.9 \%$ (95\% CI $7.5 \%$ to $24.1 \%)$ ). In addition, the same study reported low prevalence of LA in HIV $(2 \%)$, HCV $(<1 \%)$ and hepatitis B virus (HBV) $(1 \%)$ compared with the LA prevalence in our analysis $(50.7 \%$ $(95 \%$ CI $34.8 \%$ to $66.5 \%)$ ). In other words, it looks like the predominant pattern of aPL in viral infections has shifted from aCL in HIV, HCV and EBV to LA in SARS-CoV-2. Among antiphospholipid antibodies, it is well known that presence of LA is strongly associated with thrombosis compared with aCL and anti-B2 GPI. ${ }^{32}$

However, the high prevalence of LA in this analysis must be interpreted carefully as LA testing results, in contrast to aCL and anti- $\$ 2$ GPI testing, can be affected by heparin administration and cause false positive results. ${ }^{334}$ Current guidelines ${ }^{33} 34$ recommend caution when interpreting LA testing in patients receiving heparin and recommend blood to be drawn for LA testing after 12 hours since the last dose of heparin. Unfortunately, this was not considered when interpreting LA results in all of the studies included in this meta-analysis except in one study ${ }^{22}$ Thus, heparin administration is a potential bias in our analysis; however, the three-step (dRVVT) screen test that has been used in the studies of this meta-analysis can neutralise any heparin effect, especially in prophylactic dose. ${ }^{35}$ Seventy-one per cent of this analysis subjects received prophylactic dose, while the rest received therapeutic dose, and according to British Committee for

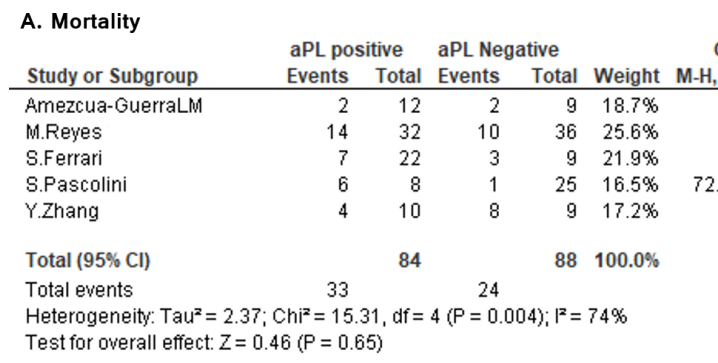

Odds Ratio Odds Ratio

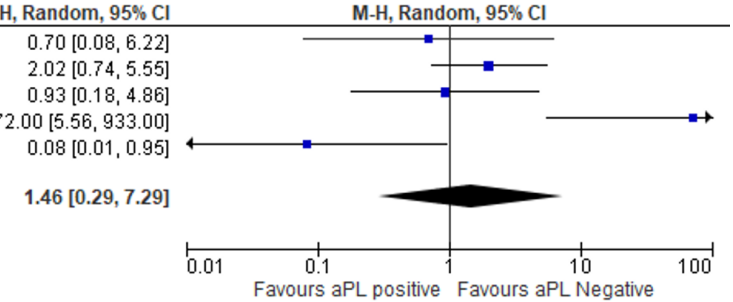

B. Invasive ventilation

\begin{tabular}{lrrrrr} 
& \multicolumn{6}{c}{ aPL positive } & \multicolumn{3}{l}{ aPL Negative } \\
Study or Subgroup & Events & Total & Events & Total & Weight \\
\hline Amezcua-GuerraLM & 7 & 12 & 5 & 9 & $21.5 \%$ \\
M.Reyes & 15 & 32 & 19 & 36 & $54.9 \%$ \\
M.Xiao & 29 & 31 & 28 & 35 & $23.6 \%$ \\
Total $(95 \% \mathrm{Cl})$ & & 75 & & 80 & $100.0 \%$ \\
Total events & 51 & & 52 & & \\
Heterogeneity: Tau $^{2}=0.12 ; \mathrm{Chi}^{2}=2.46, \mathrm{df}=2(\mathrm{P}=0.29) ; \mathrm{I}^{2}=19 \%$
\end{tabular}

Test for overall effect: $Z=0.45(P=0.65)$

Figure 4 Forest plot on the odds of mortality $(A)$ and invasive ventilation $(B)$ in aPL-positive patients with COVID-19 compared with aPL-negative patients with COVID-19. aPL, anticardiolipin antibodies. 
Venous thromboembolism

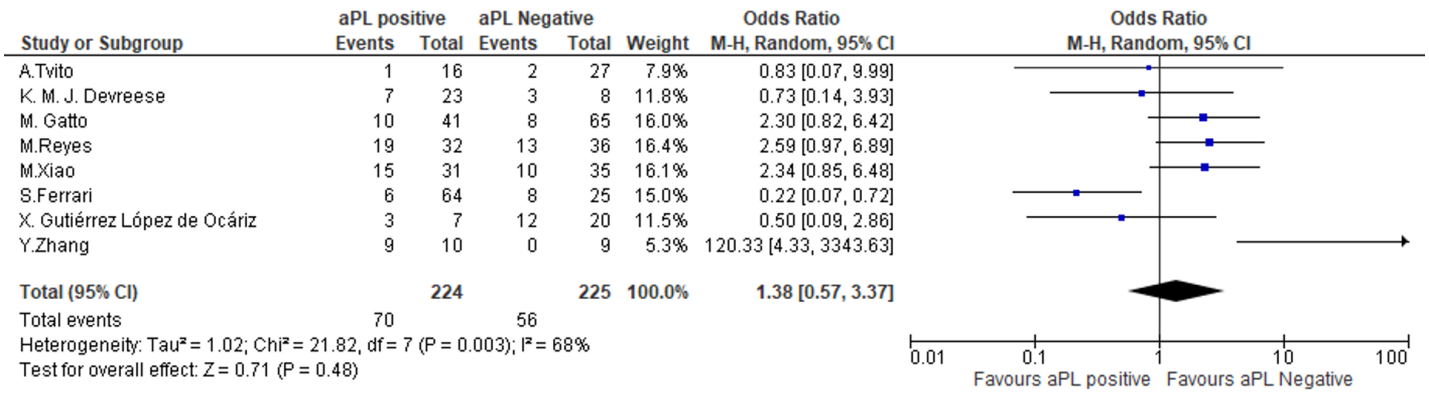

Figure 5 Forest plot on the odds of venous thromboembolism in aPL-positive patients with COVID-19 compared with aPLnegative patients with COVID-19. aPL, anticardiolipin antibodies.

Standards in Haematology guideline, ${ }^{34}$ prophylactic doses of heparin should have less effect on LA testing results. Therefore, the fact that dRVVT screening test was used in this analysis subjects and most of them received prophylactic dose of heparin may reduce the risk of bias related to heparin administration. In summary, despite the potential bias of LA testing by heparin administration, LA may be truly elevated in SARS-CoV-2 compared with other viral infections. This finding requires further investigations to examine the true prevalence and role of LA in SARS-CoV-2 infection.

One of our significant findings of this study is that severe COVID-19 disease requiring ICU care is associated with higher prevalence of aCL and anti-32 GPI. In contrast, severe disease was not found to be associated with the prevalence of LA. However, this finding might be biased by heparin administration that interfere with LA testing as explained before. High prevalence of aPL in critically ill patients has been reported previously. ${ }^{36}$ This can be partially explained by the extensive inflammation, cellular damage and apoptosis in critically ill patients that can induce aPL production. ${ }^{3738}$ aPL are antibodies targeting mainly phospholipid-binding proteins, such as $\beta 2$-GPI and prothrombin, that expressed on cell membrane at high density. ${ }^{38}$ It was hypothesised that the damaged apoptotic cell surfaces expose these cellular components to the immune system, predisposing an individual to develop aPL. ${ }^{38}$ Although aPL is associated with critical illness, this analysis as well as previous studies ${ }^{3639}$ did not find a significant association between aPL and disease outcomes like invasive ventilation and mortality. These findings suggest that aPL may be markers for disease severity or tissue injury but if aPL contribute to tissue damage and disease severity is questionable and require further investigations.

Surprisingly, the presence of aPL was not significantly associated with elevated D-dimer or thrombosis. This may be explained by the low titres or transient elevation of aPL in COVID-19 as compared with patients with antiphospholipid syndrome. ${ }^{16} 25$ Another interesting explanation is that aPL found in viral infections or healthy aPL carriers might be different and less pathogenic than the ones found in antiphospholipid syndrome. A recent study showed that healthy aPL carriers had higher anti- $\beta 2$ GPI-D4/ 5 but lower anti- $\beta 2$ GPI-D1 compared with patients with antiphospholipid syndrome. ${ }^{40}$ Anti- $\beta 2$ GPI-D1 subclass is associated with higher risk of thrombosis compared with anti- $\beta 2$ GPI-D $4 / 5 .{ }^{41}$ In addition, it

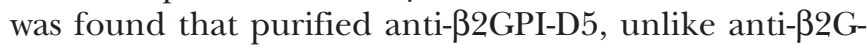
PI-D1, did not recognise cardiolipin-bound $\beta 2$ glycoprotein I while being able to interact with $\beta 2$ glycoprotein. ${ }^{42}$ This might explain the higher prevalence of anti-32 GPI compared with aCL reported in some of the studies included in this analysis. ${ }^{15} 161820$ To our knowledge, none of the studies subclassified IgG aPL in patients with COVID-19; therefore, subclassification of IgG aPL in COVID-19 is needed.

Although we found that the presence of aPL was not significantly associated with thrombosis, a direct relationship between LA alone and thrombosis has not been studied. Also, none of the studies examined the relationship between combined positivity for aPL and thrombosis knowing that combined positivity for aPL carries higher risk of thrombosis. ${ }^{32}$ Additionally, heparin was administered to most of the subjects included in this meta-analysis at either prophylactic or therapeutic dose. Heparin administration may have decreased macrothrombosis and confounded the association between aPL and thrombosis in patients with COVID-19. In contrast to macrothrombosis, the direct measurement of microangiopathy and microthrombi is difficult in living organisms. Microthrombi in the small lung vasculatures is a common microscopic finding, occurring in $80 \%-100 \%$ of lungs examined at autopsy. ${ }^{4}$ Thus, despite the lack of association between the presence of aPL and macrothrombosis, the presence of aPL may have contributed to the widespread microvascular thrombosis seen in patients with COVID-19. ${ }^{34}$

In summary, aPL were frequent in patients with COVID19 , and severe disease is associated with higher prevalence of aPL. Although our analysis showed that the presence of aPL was not associated with thrombosis, invasive ventilation and mortality, these findings still cannot rule out any contribution of aPL in the pathogenesis of COVID-19.

\section{CONCLUSION}

Based on this meta-analysis, we concluded that the prevalence of aPL is high in patients with COVID-19, and LA 
is the most frequent aPL reported. The prevalence of aPL was higher in ICU compared with non-ICU patients. Our analysis found no correlation between aPL positivity and disease outcomes such as thrombosis, invasive ventilation and mortality. These findings may suggest that aPL are just markers for disease severity rather than being pathogenic. However, further investigations are still required to identify the contribution of these markers in the pathogenesis of COVID-19.

\section{LIMITATION}

Due to meta-analysis nature, our results have several limitations: (1) as discussed previously, thrombotic events reported in some studies without mentioning if the prophylactic or therapeutic heparin were administered. (2) Heparin administration and elevated CRP may impact the LA testing results. (3) Most studies did not mention when aPL was measured during hospitalisation. (5) Not all studies excluded patients with previous history of autoimmune diseases or thrombophilia. (6) Most studies used the value $>20 \mathrm{U}$ as a cut-off value for positive result; however, several others ${ }^{913-15} 181922$ did not specify the exact cut-off value. This cut-off value is apparently low, and likely borderline results were considered positive. Low aPL titres might have low prognostic values, whereas the higher aPL titre $(>40 \mathrm{U})$ is well known to carry higher risk for thrombosis and is required for the diagnosis of antiphospholipid syndrome.

Contributors MT: collecting data, analysis and writing manuscript. LS: collecting data, analysis, writing manuscript and correction.

Funding The work was supported by a grant by NHLBI (R01HL150474) to LS. Competing interests None declared.

Patient consent for publication Not required.

Provenance and peer review Not commissioned; externally peer reviewed.

Data availability statement Data are available in a public, open access repository.

Open access This is an open access article distributed in accordance with the Creative Commons Attribution Non Commercial (CC BY-NC 4.0) license, which permits others to distribute, remix, adapt, build upon this work non-commercially, and license their derivative works on different terms, provided the original work is properly cited, appropriate credit is given, any changes made indicated, and the use is non-commercial. See: http://creativecommons.org/licenses/by-nc/4.0/.

ORCID iD

Muhanad Taha http://orcid.org/0000-0002-5867-1929

\section{REFERENCES}

1 Bilaloglu S, Aphinyanaphongs Y, Jones S, et al. Thrombosis in hospitalized patients with COVID-19 in a new York City health system. JAMA 2020;324:799-801.

2 Zhang Y, Xiao M, Zhang S, et al. Coagulopathy and antiphospholipid antibodies in patients with Covid-19. N Engl J Med Overseas Ed 2020;382:e38.

3 Maiese A, Manetti AC, La Russa R, et al. Autopsy findings in COVID19-related deaths: a literature review. Forensic Sci Med Pathol 2020. doi:10.1007/s12024-020-00310-8. [Epub ahead of print: 07 Oct 2020].

4 Carsana L, Sonzogni A, Nasr A, et al. Pulmonary post-mortem findings in a series of COVID-19 cases from northern Italy: a twocentre descriptive study. Lancet Infect Dis 2020;20:1135-40.

5 Connors JM, Levy JH. Thromboinflammation and the hypercoagulability of COVID-19. J Thromb Haemost 2020.
6 Panigada M, Bottino N, Tagliabue P, et al. Hypercoagulability of COVID-19 patients in intensive care unit: a report of thromboelastography findings and other parameters of hemostasis. J Thromb Haemost 2020;18:1738-42.

7 Amezcua-Guerra LM, Rojas-Velasco G, Brianza-Padilla M, et al. Presence of antiphospholipid antibodies in COVID-19: case series study. Ann Rheum Dis 2020. doi:10.1136/ annrheumdis-2020-218100. [Epub ahead of print: 04 Aug 2020].

8 Gatto M, Perricone C, Tonello M, et al. Frequency and clinical correlates of antiphospholipid antibodies arising in patients with SARS-CoV-2 infection: findings from a multicentre study on 122 cases. Clin Exp Rheumatol 2020;38:754-9.

9 Pineton de Chambrun M, Frere C, Miyara M. High frequency of antiphospholipid antibodies in critically ill COVID-19 patients: a link with hypercoagulability? J Intern Med 2020.

10 Previtali G, Seghezzi M, Moioli V, et al. The pathogenesis of thromboembolic disease in covid-19 patients: could be a catastrophic antiphospholipid syndrome? Thromb Res 2020;194:192-4.

11 Reyes Gil M, Barouqa M, Szymanski J, et al. Assessment of lupus anticoagulant positivity in patients with coronavirus disease 2019 (COVID-19). JAMA Netw Open 2020;3:e2017539.

12 Siguret V, Voicu S, Neuwirth M, et al. Are antiphospholipid antibodies associated with thrombotic complications in critically ill COVID-19 patients? Thromb Res 2020;195:74-6.

13 Pascolini S, Vannini A, Deleonardi G, et al. COVID-19 and immunological dysregulation: can autoantibodies be useful? Clin Trans/ Sci 2021;14:502-8.

14 Tvito A, Ben-Chetrit E, Zimmerman FS, et al. Lupus anticoagulant in patients with COVID-19. Int J Lab Hematol 2021;43:e17-e18.

15 Vlachoyiannopoulos PG, Magira E, Alexopoulos $\mathrm{H}$, et al. Autoantibodies related to systemic autoimmune rheumatic diseases in severely ill patients with COVID-19. Ann Rheum Dis 2020;79:1661-3.

16 Xiao M, Zhang Y, Zhang S. Antiphospholipid antibodies in critically ill patients with COVID-19. Arthritis Rheumatol 2020.

17 Zuo Y, Estes SK, Ali RA, et al. Prothrombotic autoantibodies in serum from patients hospitalized with COVID-19. Sci Trans/ Med 2020;12. doi:10.1126/scitransImed.abd3876. [Epub ahead of print: 1811 2020].

18 Borghi MO, Beltagy A, Garrafa E, et al. Prevalence, specificity, and clinical association of anti-phospholipid antibodies in COVID-19 patients: are the antibodies really guilty? medRxiv 2020. doi:10.1101 /2020.06.17.20134114. [Epub ahead of print: 19 Jun 2020].

19 Fan S, Xiao M, Han F, et al. Neurological manifestations in critically ill patients with COVID-19: a retrospective study. Front Neurol 2020;11.

20 Ferrari E, Sartre B, Squara F, et al. High prevalence of acquired thrombophilia without prognosis value in patients with coronavirus disease 2019. J Am Heart Assoc 2020;9:e017773.

21 Gazzaruso C, Carlo Stella N, Mariani G, et al. High prevalence of antinuclear antibodies and lupus anticoagulant in patients hospitalized for SARS-CoV2 pneumonia. Clin Rheumatol 2020;39:2095-7.

22 Guerrero EV. Thrombosis and antiphospholipid antibodies in patients with SARS-COV-2 infection (COVID-19). Int J Lab Hematol 2020.

23 Harzallah I, Debliquis A, Drénou B. Lupus anticoagulant is frequent in patients with Covid-19. J Thromb Haemost 2020.

24 Helms J, Tacquard C, Severac F, et al. High risk of thrombosis in patients with severe SARS-CoV-2 infection: a multicenter prospective cohort study. Intensive Care Med 2020;46:1089-98.

25 Devreese KMJ, Linskens EA, Benoit D, et al. Antiphospholipid antibodies in patients with COVID-19: a relevant observation? $\mathrm{J}$ Thromb Haemost 2020;18:2191-201.

26 Bertin D, Brodovitch A, Beziane A, et al. Anticardiolipin IgG autoantibody level is an independent risk factor for COVID-19 severity. Arthritis Rheumatol 2020;72:1953-5.

27 Moher D, Liberati A, Tetzlaff J, et al. Preferred reporting items for systematic reviews and meta-analyses: the PRISMA statement. PLoS Med 2009;6:e1000097.

28 Xiao M, Zhang Y, Zhang S. Brief report: Anti-phospholipid antibodies in critically ill patients with coronavirus disease 2019 (COVID-19). Arthritis Rheumatol 2020;72:1998-2004.

29 Zuo Y, Estes S, Ali R. Prothrombotic antiphospholipid antibodies in COVID-19. medRxiv Prepr Serv Heal Sci 2020.

30 Uthman IW, Gharavi AE. Viral infections and antiphospholipid antibodies. Semin Arthritis Rheum 2002;31:256-63.

31 Abdel-Wahab N, Talathi S, Lopez-Olivo MA, et al. Risk of developing antiphospholipid antibodies following viral infection: a systematic review and meta-analysis. Lupus 2018;27:572-83.

32 Galli M, Luciani D, Bertolini G, et al. Lupus anticoagulants are stronger risk factors for thrombosis than anticardiolipin antibodies in 
the antiphospholipid syndrome: a systematic review of the literature. Blood 2003;101:1827-32.

33 Pengo V, Tripodi A, Reber G, et al. Update of the guidelines for lupus anticoagulant detection. J Thromb Haemost 2009;7:1737-40.

34 Keeling D, Mackie I, Moore GW, et al. Guidelines on the investigation and management of antiphospholipid syndrome. Br J Haematol 2012;157:47-58.

35 De Kesel PMM, Devreese KMJ. The effect of unfractionated heparin, enoxaparin, and danaparoid on lupus anticoagulant testing: can activated carbon eliminate false-positive results? Res Pract Thromb Haemost 2020;4:161-8.

36 Vassalo J, Spector N, Meis Ede, et al. Antiphospholipid antibodies in critically ill patients. Rev Bras Ter Intensiva 2014;26:176-82.

37 Asherson RA, Cervera R. Antiphospholipid antibodies and infections. Ann Rheum Dis 2003;62:388-93.

38 Giannakopoulos B, Passam F, Rahgozar S, et al. Current concepts on the pathogenesis of the antiphospholipid syndrome. Blood 2007;109:422-30.
39 Vassalo J, Spector N, de Meis E, et al. Antiphospholipid antibodies in critically ill patients with cancer: a prospective cohort study. J Crit Care 2014;29:533-8.

40 Andreoli L, Chighizola CB, Nalli C, et al. Clinical characterization of antiphospholipid syndrome by detection of IgG antibodies against $\beta 2$-glycoprotein I domain 1 and domain 4/5: ratio of anti-domain 1 to anti-domain $4 / 5$ as a useful new biomarker for antiphospholipid syndrome. Arthritis Rheumatol 2015;67:2196-204.

41 Liu T, Gu J, Wan L. Anti- $\beta 2$ GPI domain 1 antibodies stratify high risk of thrombosis and late pregnancy morbidity in a large cohort of Chinese patients with antiphospholipid syndrome. Thromb Res 2020.

42 Durigutto P, Grossi C, Borghi MO, et al. New insight into antiphospholipid syndrome: antibodies to $\beta 2$ glycoprotein I-domain 5 fail to induce thrombi in rats. Haematologica 2019;104:819-26. 